\title{
Identification of two novel mutations in the PLCD1 gene in Chinese patients with hereditary leukonychia
}

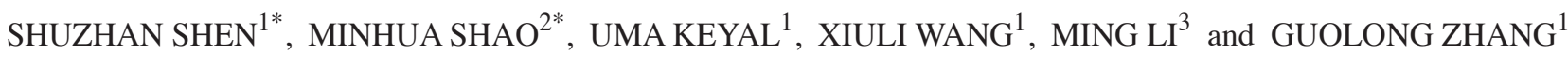 \\ ${ }^{1}$ Institute of Photomedicine, Shanghai Skin Disease Hospital, Tongji University School of Medicine, \\ Shanghai 200443; ${ }^{2}$ Department of Dermatology, Affiliated Wuxi Children's Hospital, \\ Nanjing Medical University, Wuxi, Jiangsu 214023; ${ }^{3}$ Department of Dermatology, Xinhua Hospital, \\ Shanghai Jiaotong University School of Medicine, Shanghai 200092, P.R. China
}

Received February 1, 2020; Accepted March 11, 2021

DOI: $10.3892 / \mathrm{mmr} .2021 .12052$

\begin{abstract}
Hereditary leukonychia (HL) is a rare nail dystrophy disease, and several different clinical manifestations and mutations in the phospholipase $\mathrm{C} \delta 1$ (PLCDI) gene have been reported. The present study reports on one Chinese family and one sporadic case of with HL. The family members exhibited an autosomal dominant pattern of inheritance with the involvement of all the fingers and toenails in all the patients. Of interest, most of the affected members had koilonychia during their childhood. Thus, the present study first used gene mapping with an aim to identify the pathogenic gene underlying koilonychia. Through genome-wide linkage analysis, the pathogenic area of koilonychia was identified on chromosome 3 with multipoint Log of Odds scores $>2$. A novel pathogenic mutation c.1384G $>$ A (p.E462K) was identified in the PLCD1 gene in all the patients in the family, which confirmed the diagnosis of hereditary leukonychia. A novel mutation c.770G>A (p.R257H) was also detected in one sporadic case of leukonychia. On the basis of these findings and of previous studies, it is suggested that hereditary leukonychia may initially present as koilonychia, whereas hereditary koilonychia does not progress to leukonychia. Moreover, the present study identified two pathogenic variants of the PLCD1 associated with hereditary leukonychia, and highlights the significance of genetic diagnosis.
\end{abstract}

Correspondence to: Professor Guolong Zhang, Institute of Photomedicine, Shanghai Skin Disease Hospital, Tongji University School of Medicine, 1278 Baode Road, Jingan, Shanghai 200443, P.R. China

E-mail: zglamu@163.com

Professor Ming Li, Department of Dermatology, Xinhua Hospital, Shanghai Jiaotong University School of Medicine, 1665 Kongjiang Road, Yangpu, Shanghai 200092, P.R. China

E-mail: aypyslm@163.com

${ }^{*}$ Contributed equally

Key words: hereditary leukonychia, koilonychia, mutations, phospholipase C $\delta 1$ gene

\section{Introduction}

Leukonychia is defined by a white discoloration of the nail plate. The pathophysiology of the disease is not yet fully understood; however, it can be classified into three categories (true leukonychia, apparent leukonychia and pseudoleukonychia) based on the site of origin (1). True leukonychia can either be acquired or inherited. Acquired true leukonychia is a result of alterations in the nail matrix due to other medical conditions or external exposure (1). Hereditary leukonychia is extremely rare and it may be considered as a benign isolated occurrence or may be associated with a range of systemic diseases (2). Mutations in the phospholipase C $\delta 1$ (PLCDI) gene have been identified as a major causative factor in hereditary leukonychia (HL) (1). The PLCD1 gene maps to the short arm of chromosome 3 (3p22.2), consists of 15 exons, and the gene encodes two isoforms, 777 or 756 amino acids in length. PLCDI is the enzyme required for $\mathrm{Ca}^{2+}$ signal transduction in a number of tissues, such as the foreskin, keratinocytes, nail matrix and fibroblasts, which is significant in the manifestation of HL (3).

The present study investigated a Chinese family with HL, some of whom were diagnosed with koilonychia during their childhood, as well as a sporadic patient who presented with HL. Genome-wide linkage analysis revealed the linkage of the family to chromosome 3 and Sanger sequencing of PLCD1 gene identified one novel heterozygous missense mutation c.1384G >A (p.E462K). Moreover, another novel mutation c.770G $>$ A (p.R257H) in the sporadic case of leukonychia was detected.

\section{Case report}

Clinical characteristics of the patients. A family consisting of 6 affected and 10 unaffected individuals, and 1 sporadic patient with white-colored nails from birth were enrolled in the present study. Informed consent was obtained from all study participants prior to their inclusion in the study. The present study was approved by the Institutional Ethical Committee of Shanghai Skin Disease Hospital and was performed in accordance with the guidelines of the Declaration of Helsinki (4). The family had no consanguineous marriages and the 


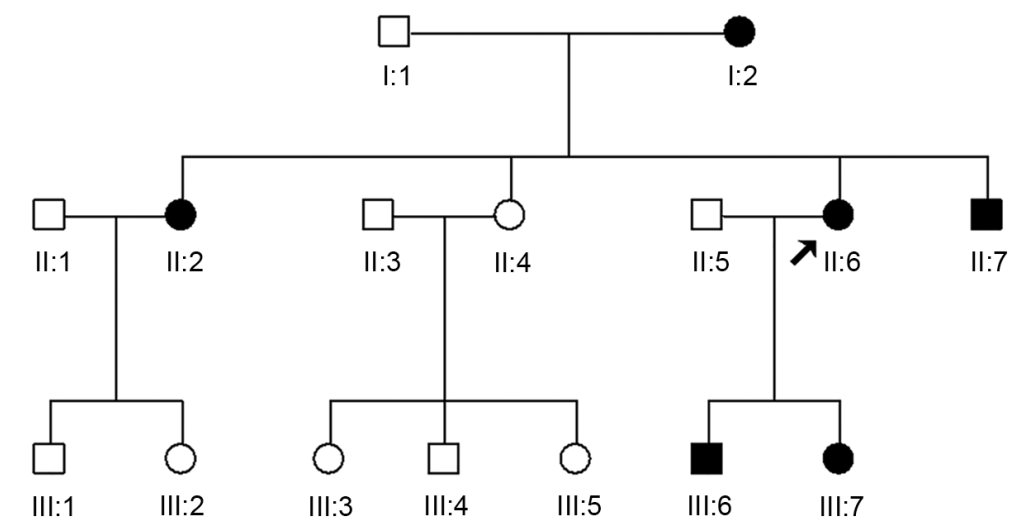

Figure 1. Pedigree of the hereditary leukonychia family. Circles and squares represent females and males, respectively. Clear symbols represent unaffected individuals, whereas filled symbols represent affected individuals.
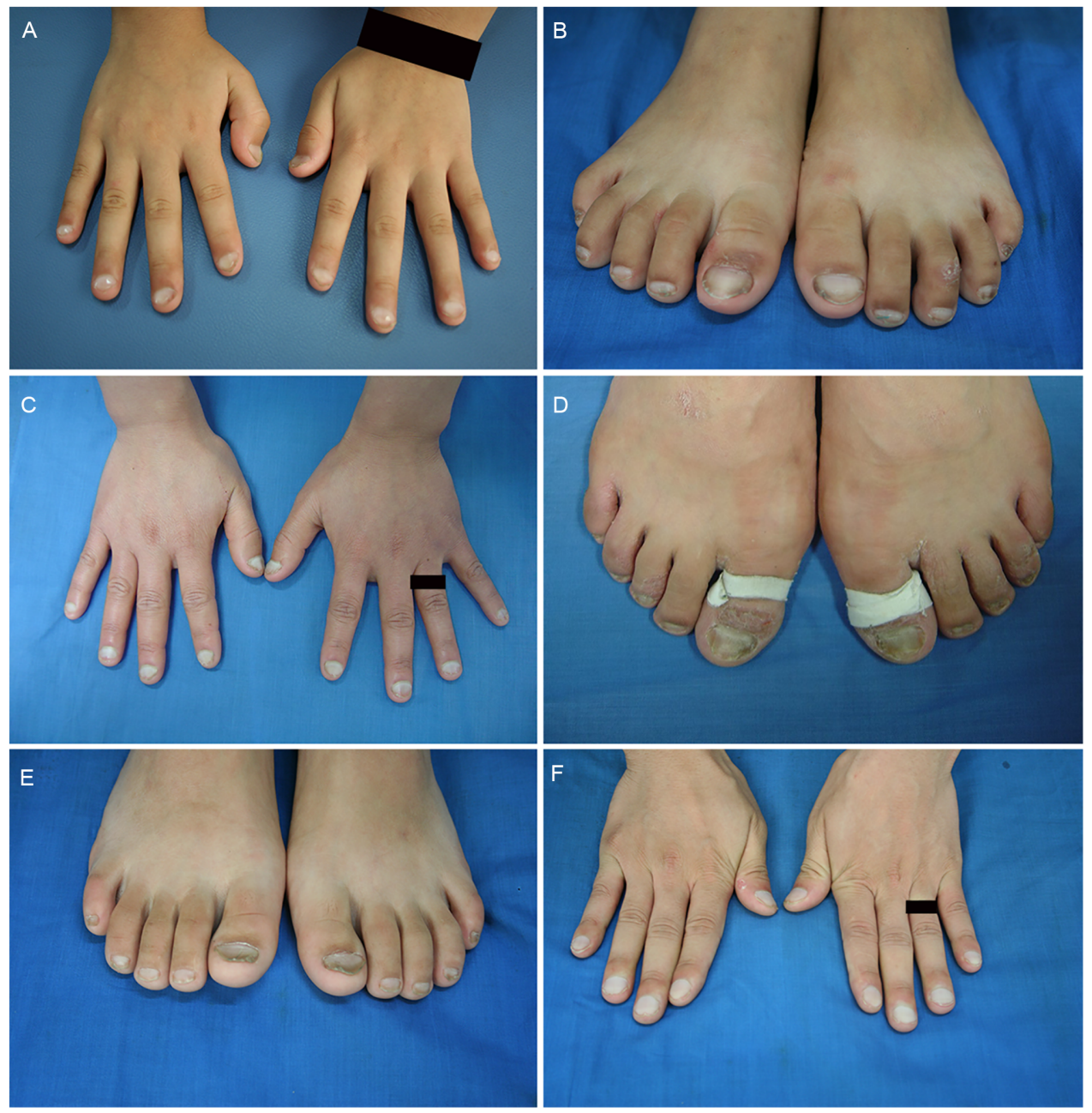

Figure 2. Clinical presentation of the family with hereditary leukonychia. (A and B) The proband had complete concave and white fingernail and toenail plates, with rough and light brown distal edges. (C-F) Leukonychia with koilonychia of the nail plate in fingernails and toenails in the affected individuals of the family.

findings were consistent with an autosomal dominant mode of inheritance of the disease (Fig. 1). The proband was an 8 -year-old boy born with white-colored soft nails. The nails became flat or spoon-shaped with the passing of time. Upon examination, his fingernails were quite thin with a concave surface, and the distal edges of some of the nail plates were rough and hyperpigmented, particularly those on both thumbs . The toenails had minor lesions along with varying degrees of browning at the distal edges (Fig. 2A and B). A white discoloration of the nail plates was present since birth. Upon applying pressure over the nail plates, no fading of the whiteness was observed. A skin, hair and systemic examination revealed no 
abnormal findings. Furthermore, no evidence of any associated conditions was found. Routine laboratory test results were normal. A direct microscopy test of fungus from the fingernails and toenails yielded negative results. Serum levels of iron, zinc and $\mathrm{Ca}^{2+}$ were normal. Oral treatment with Theragran Junior did not improve the condition. Upon examination of the other members of the family, 6 patients with leukonychia, including 2 males and 4 females with similar spoon-shaped nails during their childhood (out of 15 members of 3 generations) were identified (Fig. 2C-F). The changes in the nails of all patients were similar; however, certain differences were observed. The similarities were that the spoon-shaped appearances of the nails had improved to varying degrees, and the brown color of the distal nails had a tendency of centripetal growth with age. The differences lied in the appearance of the individual patients' nails. The nails of one of the affected members (II:7) only exhibited spoon-shaped changes without browning during childhood, and they became normal during adulthood. However, the browning of the proband's nails was more evident.

Linkage of hereditary leukonychia to chromosome 3. Owing to the typical features of koilonychia in the patients in the present study, an Illumina Infinium HumanLinkage-24 panel (Illumina, Inc.) was used to genotype 12 individuals from the family (I:1, I:2, II:1, II:2, II:4, II:5, II:6, II:7, III:1, III:2, III:6 and III:7) (Fig. 3). Genome-wide linkage analysis was performed with a total of 6,518 single nucleotide polymorphisms (SNP) markers; their average genetic and physical distances were $442 \mathrm{~kb}$ and $0.55 \mathrm{cM}$. In the linkage analysis, SNPs with a call rate $<90 \%$, monomorphic SNPs and non-Mendelian transmitted markers were removed; a total number of 45,716 informative autosomal SNPs remained. Multipoint parametric linkage analyses were performed with the MERLIN program version 1.1.2 (5). A fully penetrant autosomal-dominant model was used with a rare disease frequency of 0.0001 . Critical recombination events of the pedigree members were also determined through haplotype construction in MERLIN.

Genome-wide linkage analysis in the family revealed that the multipoint Log of Odds (LOD) scores of the third chromosome were $>2.0$, indicating linkages to this chromosome. No other locus suggestive of linkage was detected. Recombination events were observed in individuals II:3 and III:1. These recombination events defined that the physical distance of the susceptibility region was a $28.56 \mathrm{cM}$ interval (54.98-83.54 cM between kgp3166993 and rs6445345). All affected individuals shared a common haplotype across this disease interval.

Sequencing analysis. One known pathogenic gene for leukonychia was located in the mapped region (6). Primers were designed for all 15 coding exons of PLCD1 (RefSeq accession no. NM_006225). The QIAquick PCR Purification kit (Qiagen, Inc.) was used to purify the PCR products and PLCDI was sequenced with an ABI PRISM 3730 automated sequencer (Applied Biosystems; Thermo Fisher Scientific, Inc.). Sequence comparisons and analysis were performed using the Phred-Phrap-Consed program version 12.0 (5). A total of 10 templates were sequenced per subject. The accession

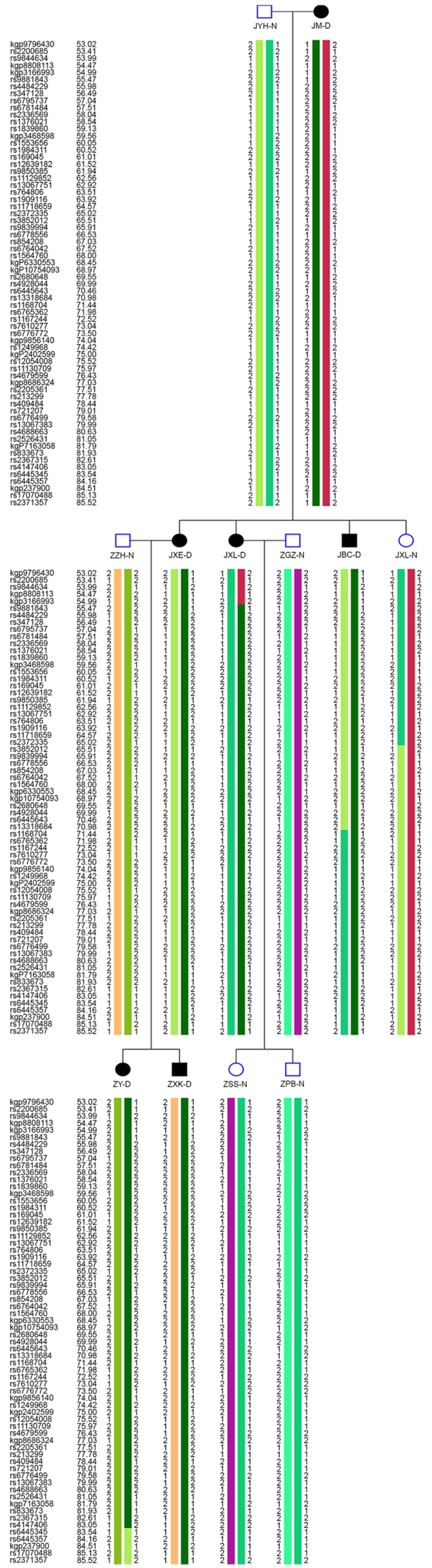

Figure 3. Linkage analysis of the family with hereditary leukonychia. 
A 500

C

470

480

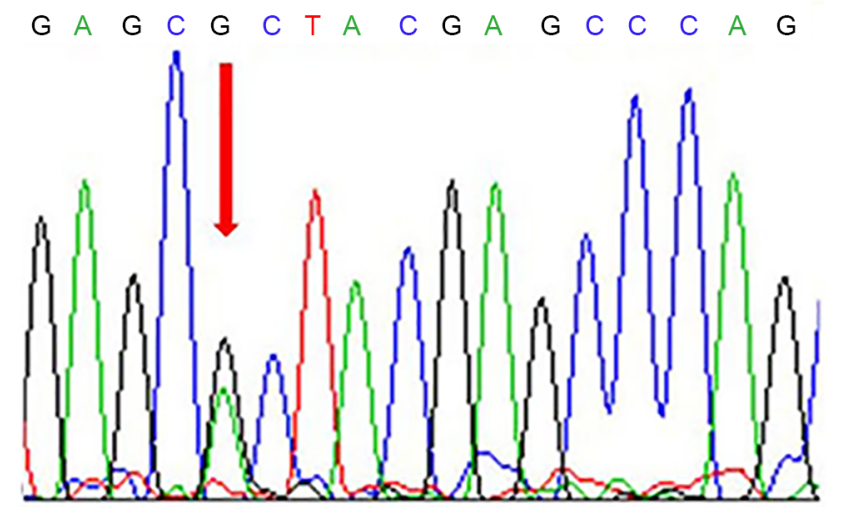

$\begin{array}{ccccccccccccccc}T & C & A & G & A & C & A & A & A & G & A & C & G & A & G\end{array}$

B

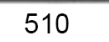

D
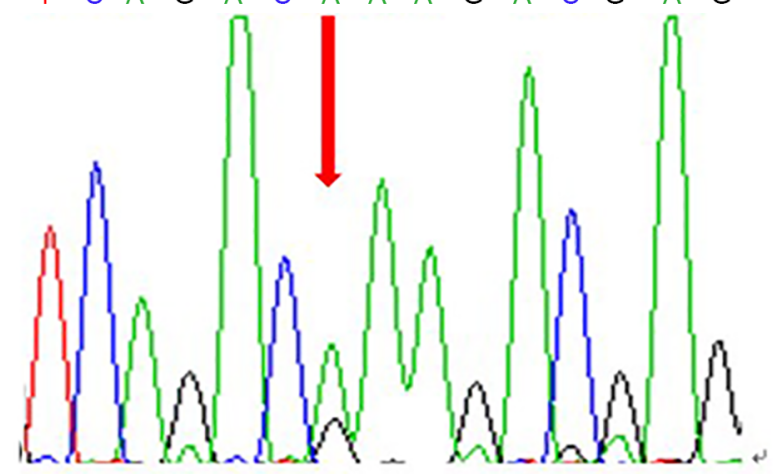

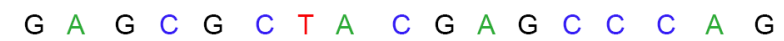

470

480

$T C A \quad G \quad A \quad C \quad G \quad A \quad A \quad G \quad A \quad C \quad G \quad A \quad G \quad G$
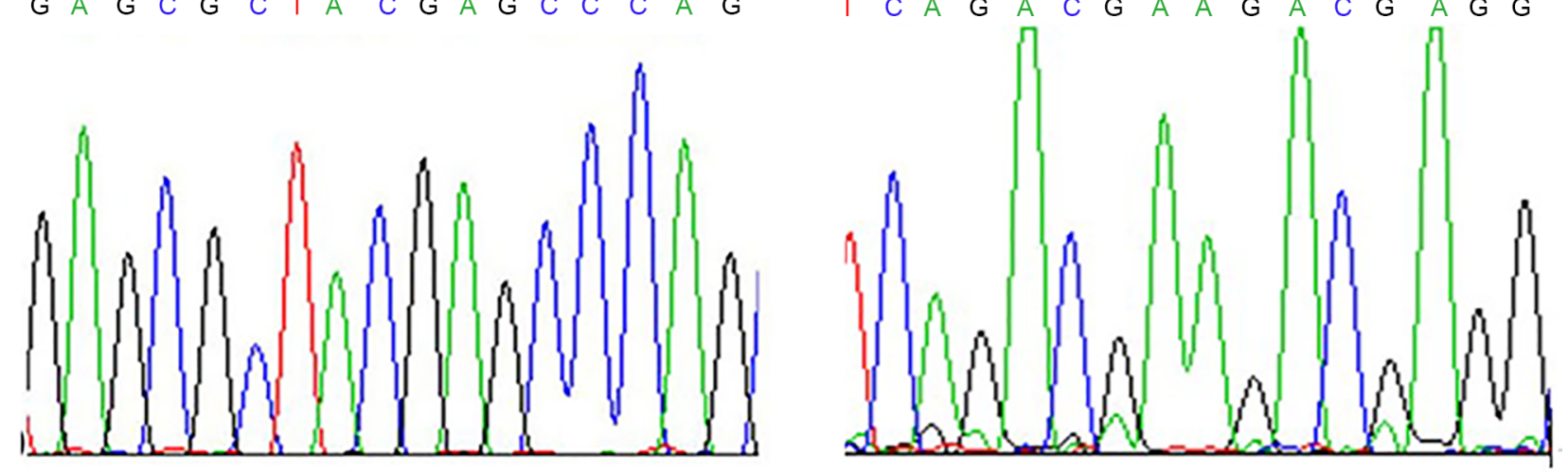

E

$\begin{array}{ll}\text { Human } & \text { PAGGENGPEATDVSDEDEAAEMEDEAVRSQV } \\ \text { Mouse } & \text { PAGGENGPEATDVSDEDEAAEMEDEAVRSQV } \\ \text { Dog } & \text { PAGGENGPEATDVSDEDEAAEMEDEAVRSQV } \\ \text { Chicken } & \text { PAGGENGPEATDVSDEDEAAEMEDEAVRSQV } \\ \text { Chimp } & \text { PAGGENGPEATDVSDEDEAAEMEDEAVRSQV } \\ \text { Cattle } & \text { PAGGENGPEATDVSDEDEAAEMEDEAVRSQV } \\ \text { Zebrafish } & \text { PAGGENGPEATDVSDEDEAAEMEDEAVRSQV } \\ \text { Horse } & \text { PAGGENGPEATDVSDEDEAAEMEDEAVRSQV } \\ \text { Pig } & \text { PAGGENGPEATDVSDEDEAAEMEDEAVRSQV } \\ \text { Goat } & \text { PAGGENGPEATDVSDEPEAAEMEDEAVRSQV }\end{array}$

REEEAGPALALSLIEPYEPSETAKAQRQMTK
REEEAGPALALSLIERYEPSETAKAQRQMTK
REEEAGPALALSLIERYEPSETAKAQRQMTK
REEEAGPALALSLIERYEPSETAKAQRQMTK
REEEAGPALALSLIERYEPSETAKAQRQMTK
REEEAGPALALSLIERYEPSETAKAQRQMTK
REEEAGPALALSLIERYEPSETAKAQRQMTK
REEEAGPALALSLIERYEPSETAKAQRQMTK
REEEAGPALALSLIERYEPSETAKAQRQMTK
REEEAGPALALSLIERYEPSETAKAQRQMTK

Figure 4. Sequencing results of the PLCD1 gene. (A) The novel missense mutation c.770G>A in exon 5 of the $P L C D 1$ gene (red arrow) was confirmed by Sanger sequencing in the sporadic leukonychia patient. (B) The wild-type sequence from an unaffected member. (C) A novel missense mutation c.1384G $>A$ in exon 9 of the PLCD1 gene (red arrow) was found in the proband of the hereditary leukonychia family by Sanger sequencing. (D) A wild-type sequence from an unaffected member of the family. (E) Mutations p.E462K and p.R257H are conserved in all species compared in the figure. All data were cited from //asia.ensembl.org/index.html.

number of the reference sequence used for the identification of mutations/SNPs is NM_001130964.2. The software used for mutation/SNP analysis in the present study was Chromas 233 (5). A total of 100 unrelated population-matched control peripheral blood samples were sequenced to exclude the possibility of identification of the polymorphism in the PLCDI gene. The unrelated matched control samples used in the study were collected from volunteers who had come to the hospital for physical examination between January and March in 2013. The median age of 100 unrelated population was 37.5. The age range was 23-61. Among them, 58 individuals were male and 42 individuals were female. All data were cited from //asia.ensembl.org/index.html.
Identification of mutations in the PLCDI gene. Direct sequencing of $P L C D I$ revealed a heterozygous missense mutation involving a $\mathrm{G}$ to $\mathrm{A}$ transition at nucleotide position 1384 (c.1384G $>$ A) of the gene in the Chinese pedigree patients (Fig. 4C and D). This resulted in the substitution of a codon for glutamic acid at amino acid position 462 to lysine (p.E462K). The other heterozygous missense mutation detected in exon 5 in one sporadic case of leukonychia was a $\mathrm{G}$ to $\mathrm{A}$ transition at nucleotide 770 (c.770 G>A) (Fig. 4A and B), which resulted in the substitution of a codon for arginine at amino acid position 257 to histidine (p.R257H). To exclude the possibility that the two mutations do not represent non-pathogenic polymorphisms, the Polyphen2 (polymorphism phenotyping version 2 
(genetics.bwh.harvard.edu/pph2) tool was used to assess the effects of these variations on the function of the corresponding protein. It was found that the $\mathrm{c} .1384 \mathrm{G}>\mathrm{A}$ and c. $770 \mathrm{G}>\mathrm{A}$ mutations in the PLCD1 gene were both pathogenic. The Polyphen2 score for the c.1384G $>$ A mutation was 0.984 (close to 1), while that for c.770 G>A was 0.885 (close to 1). PolyPhen scores were interpreted as follows: Benign, 0.00 to 0.20 ; possibly damaging, 0.20 to 0.85 ; and probably damaging, 0.85 to 1.00 . In addition, a panel of 200 unaffected, unrelated and ethnically matched control individuals were screened, and these mutations were not identified. These findings indicate that both mutations are not SNPs. The mutation p.E462K detected in the Chinese pedigree patients and p.R257H detected in the sporadic case of leukonychia have been shown to be highly conserved during evolution amongst dog, zebrafish, horse, mouse, chicken, chimp, cattle, pig and goat (Fig. 4E), and may be critical for the function of the protein.

\section{Discussion}

HL is a rare nail pathology that causes partial or total whitening of the nail plate. Histological analysis indicates hyperkeratosis and parakeratosis from the nail bed to the nail plate, and abnormal cornified cells in the nail plate $(1,7)$. In the present study, the clinical manifestations of the Chinese HL family were not particularly typical, with some patients presenting with unnoticeable leukonychia, but obvious koilonychia. Detailed analysis revealed that all the affected members had koilonychia in their childhood. This had misled the initial diagnosis as hereditary koilonychia and genome-wide linkage analysis was used to identify the pathogenic genes of koilonychia. Interestingly, it was revealed that the multipoint LOD scores of the third chromosome were $>2.0$, indicating linkages to this chromosome. One known pathogenic gene for leukonychia, $P L C D 1$, is present in this chromosome (8). A novel heterozygous missense mutation in the PLCDI gene was then identified by direct sequencing, which suggested that the accurate diagnosis should be hereditary leukonychia. Another mutation in the PLCDI gene was also identified in a sporadic case, which confirmed the final diagnosis.

After reviewing the literature related to hereditary leukonychia, it was found that mutations in five genes are linked to the pathogenesis of HL (9). Different genes may be associated with distinct clinical phenotypes of HL. In particular, mutations in $P L C D 1$ are predominantly reported in a large fraction of patients with HL. The PLCD1 gene consists of 15 exons, spanning $22.17 \mathrm{~kb}$ of genomic DNA on chromosome 3p22.2. PLCD1 encodes an enzyme that mediates the regulatory signaling of energy metabolism, $\mathrm{Ca}^{2+}$ homeostasis and intracellular movement $(3,10,11)$. Kiuru et al (8) discovered five mutations in the PLCDI on chromosome $3 \mathrm{p} 22.2$ in both autosomal dominant and autosomal recessive HL. Of these, three mutations, c.1309C $>$ T (p.R437X), c.1792-10del-TGTAGTGGCC and c.1055G $>$ A (p.A285GfsX70), underlie the autosomal recessive form of leuconychia, whereas the other two mutations, c.1720C >T (p.A574T) and c.625T >C (p.C209R), are responsible for the autosomal dominant form of the disease. It appears that the recessive form is caused by a premature termination codon mutation, and the autosomal dominant form by a missense mutation; one possibility would be that the missense mutations exert a dominant-negative effect on the wild-type allele with a complete loss of function. Previously, Nomikos et al (12) determined significant divergent enzymatic activities for leukonychia-linked mutant PLCD1, confirming that mutations in PLCDI cause HL. In the present study, two novel heterozygous missense mutations, c.1384G $>\mathrm{A}$ (p.E462K) and c.770 G>A (p.R257H), were identified in the family with HL and one sporadic patient, respectively. Both of the mutations are pathogenic and lead to the substitution of amino acids, which was also confirmed by Polyphen2 ( 0.984 for c. $1384 \mathrm{G}>\mathrm{A}$ and 0.885 for c.770 G>A). Moreover, it was found that the clinical manifestations of leukonychia concomitant koilonychias did not merely occur in the family described herein. The condition has also been reported in few cases of Chinese and Pakistani families (9,13-15). Thus, this phenomena may not be a result of ethnic differences.

As per current knowledge, familial koilonychia is another type of rare genodermatosis, which differs completely from HL (15). To the best of our knowledge, there are no reports of hereditary koilonychia with leukonychia to date. It is inherited in an autosomal dominant pattern with no predilection for sex. It may present at birth or within the first few years of life. Nails are typically thin and flat, developing degrees of concavity over time. In young children, koilonychia of the toenails is commonly transient and idiopathic (7). A previous literature review revealed that mutations in PLCDI only cause leukonychia (16). Although it has been proven through several proteomic studies that PLCDI is abundant in the human nail matrix, the mechanisms by which PLCD1 mutations result in leukonychia remain unknown (17), and the lack of functional verification in the present study may be considered a limitation. The present study was not able to determine whether the PLCDI gene was involved in the occurrence of hereditary koilonychia. There are no studies which have identified any pathogenic genes associated with familial koilonychia. Moreover, a family of 5 generations with a syndrome of leukonychia, koilonychia and multiple pilar cysts segregating as an autosomal dominant trait was reported by Mutoh et al (18). No specific pathogenic genes associated with the aforementioned syndrome were identified however. The syndrome was ruled out since the family presented herein did not have any other comorbidities.

In summary, HL is an extremely rare condition with multiple clinical manifestations. The present study describes a Chinese family with atypical characteristics of HL. The present study not only provides novel insights for the accurate diagnosis of HL and familial koilonychia, but also highlights the crucial significance of genetic diagnosis. Furthermore, the findings further confirm that mutations in PLCDI underlie $\mathrm{HL}$ and add another two pathogenic variants to the PLCDI mutation spectrum causing leukonychia, particularly in a Chinese Han pedigree.

\section{Acknowledgements}

Not applicable.

\section{Funding}

This study was funded by grants from National Natural Science Foundation of China (grant nos. 81471930 and 81472796). 


\section{Availability of data and materials}

The raw Sanger sequencing data have been deposited at China Nucleotide Sequence Archive (accession no. CNP0001358). It is a usual practice in our group that the data is not published for 2 years. The datasets used during the present study are available from the corresponding author upon reasonable request.

\section{Authors' contributions}

SS, MS and GZ designed the study, wrote the protocol, collected the data, performed statistical analyses and contributed to writing the manuscript. UK helped with data collection, study design and coordinated the study. ML and XW participated in the study design and helped to critically revise the manuscript. SS and GZ were responsible for confirming the authenticity of the raw data. All authors read and approved the final manuscript.

\section{Ethics approval and consent to participate}

Informed consent was obtained from all study participants prior to their inclusion in the study. The present study was approved by the Institutional Ethical Committee of Shanghai Skin Disease Hospital and was performed in accordance with the guidelines of the Declaration of Helsinki.

\section{Patient consent for publication}

Not applicable.

\section{Competing interests}

The authors declare that they have no competing interests.

\section{References}

1. Pakornphadungsit K, Suchonwanit P, Sriphojanart T and Chayavichitsilp P: Hereditary leukonychia totalis: A case report and review of the literature. Case Rep Dermatol 10: 82-88, 2018.

2. De D and Handa S: Hereditary leukonychia totalis. Indian J Dermatol Venereol Leprol 73: 355-357, 2007.

3. Kim H, Kim JY, Kim KA, Lim Y, Kim YH, Huh PW, Lee KH, Han H, Wang YP and Rha HK: Identification of the elements regulating the expression of the phospholipase $\mathrm{C}$ delta1. Mol Cells 14: 29-34, 2002.

4. Gensure RC, Mäkitie O, Barclay C, Chan C, Depalma SR, Bastepe M, Abuzahra H, Couper R, Mundlos S, Sillence D, et al: A novel COL1A1 mutation in infantile cortical hyperostosis (Caffey disease) expands the spectrum of collagen-related disorders. J Clin Invest 115: 1250-1257, 2005.
5. Zhong W, Pan Y, Shao Y, Yang Y, Yu B and Lin Z: Atypical presentation of dyschromatosis universalis hereditaria with a novel ABCB6 mutation. Clin Exp Dermatol 44: e58-e60, 2019.

6. Mir H, Khan S, Arif MS, Ali G, Wali A, Ansar M and Ahmad W: Mutations in the gene phospholipase C, delta-1 (PLCD1) underlying hereditary leukonychia. Eur J Dermatol 22: 736-739, 2012.

7. Fawcett RS, Linford S and Stulberg DL: Nail abnormalities: Clues to systemic disease. Am Fam Physician 69: 1417-1424, 2004.

8. Kiuru M, Kurban M, Itoh M, Petukhova L, Shimomura Y, Wajid M and Christiano AM: Hereditary leukonychia, or porcelain nails, resulting from mutations in PLCD1. Am J Human Genetics 88: 839-844, 2011.

9. Xue K, Zheng Y, Shen C and Cui Y: Identification of a novel PLCD1 mutation in Chinese Han pedigree with hereditary leukonychia and koilonychia. J Cosmet Dermatol 18: 912-915, 2019.

10. Song JJ, Liu Q, Li Y, Yang ZS, Yang L, Xiang TX, Ren GS and Chen JB: Epigenetic inactivation of PLCD1 in chronic myeloid leukemia. Int J Mol Med 30: 179-184, 2012.

11. Xiang T, Li L, Fan Y, Jiang Y, Ying Y, Putti TC, Tao Q and Ren G: PLCD1 is a functional tumor suppressor inducing G(2)/M arrest and frequently methylated in breast cancer. Cancer Biol Ther 10: 520-527, 2010.

12. Nomikos M, Thanassoulas A, Beck K, Theodoridou M, Kew J, Kashir J, Calver BL, Matthews E, Rizkallah P, Sideratou Z, Nounesis G and Lai FA: Mutations in PLC $\delta 1$ associated with hereditary leukonychia display divergent PIP2 hydrolytic function. FEBS J 283: 4502-4514, 2016.

13. Brown PJ, Padgett JK and English JC III: Sporadic congenital leukonychia with partial phenotype expression. Cutis 66: 117-119, 2000.

14. Khan T, Khan M, Yousaf A, Khan S, Naeem M, Shah A, Murtaza G, Ali A Jabeen N, Hussain HM, et al: Whole exome sequencing identifies a novel dominant missense mutation underlying leukonychia in a Pakistani family. J Hum Genet 63: 1071-1076, 2018.

15. Kwon NH, Kim JE, Cho BK, Jeong EG and Park HJ: Sporadic congenital leukonychia with koilonychia. Int J Dermatol 51: 1400-1402, 2012.

16. Walker J, Baran R, Vélez N and Jellinek N: Koilonychia: An update on pathophysiology, differential diagnosis and clinical relevance. J Eur Acad Dermatol Venereology 30: 1985-1991, 2016.

17. Rice RH, Xia YX, Alvarado RJ and Phinney BS: Proteomic analysis of human nail plate. J Proteome Res 9: 6752-6758, 2010.

18. Mutoh M, Niiyama S, Nishikawa S, Oharaseki T and Mukai H: A syndrome of leukonychia, koilonychia and multiple pilar cysts. Acta Derm Venereol 95: 249-250, 2015.

This work is licensed under a Creative Commons Attribution-NonCommercial-NoDerivatives 4.0 International (CC BY-NC-ND 4.0) License. 